\title{
Utility of Joint Processing Schemes
}

\author{
Annika Klockar*, Carmen Botella ${ }^{\dagger}$, Tommy Svensson ${ }^{\dagger}$, Anna Brunstrom*, Mikael Sternad ${ }^{\ddagger}$ \\ ${ }^{*}$ Computer Science, Karlstad University, Karlstad, Sweden, \{annika.klockar, anna.brunstrom\} @kau.se \\ ${ }^{\dagger}$ Signals and Systems, Chalmers University of Technology, Gothenburg, Sweden, \{carmenb, tommy.svensson\}@chalmers.se \\ ${ }^{\ddagger}$ Signals and Systems, Uppsala University, Uppsala, Sweden, mikael.sternad@ signal.uu.se
}

\begin{abstract}
Joint processing between base stations has been shown as an efficient technique to mitigate inter-cell interference and increase data rates, in particular at the cell edges. In this paper, we evaluate the utility of Internet applications in a joint processing enabled cluster of base stations. Utility is used to quantify system performance as experienced by the end user. In particular, the utility of three joint processing schemes for the downlink is characterized and compared within the cluster area. The qualitative results indicate that joint processing can significantly improve the utility of hard real-time and adaptive applications. For elastic applications, joint processing is not worthwhile, since almost as high utility is achieved also with conventional single base station assignment.
\end{abstract}

\section{INTRODUCTION}

Future wireless systems are expected to support a wide variety of services and applications with various Quality of Service (QoS) requirements. There are many metrics that can be used to evaluate a system. The ones that really count in the end are those that contribute to the performance experienced by the end user and that capture how well the system meets the requirements of its users [1]. Utility is often used to quantify the level of satisfaction and to address decision problems in networking, such as resource allocation [2], [3] and network selection for vertical handover [4], [5]. The utility of an application depends on the application performance which in turn depends on the QoS offered by the system [6]. Applications used in the Internet are often divided into the following application classes:

- Hard real-time

- Adaptive real-time

- Elastic

In conventional cellular systems, data rates are highly variable depending on the user location and hence, uniform QoS over the whole cell area becomes infeasible. Recently, coordination between base stations (BSs) is shown as a promising technique to mitigate inter-cell interference, and to increase data rates, especially for cell edge users, see for example [7], [8], [9]. Data transmission to a user is coordinated between a group of BSs, e.g. by coordinated beamforming/scheduling or joint processing/transmission. In the 3GPP standardization of LTE-Advanced these techniques are referred to as coordinated multipoint transmission/reception (CoMP) [10]. If joint processing is applied, then a user may be served by many BSs simultaneously. Joint processing over all the cellular system would introduce too large an overhead to be feasible in practice, since, e.g., channel state information (CSI) and user data would be needed in all the cooperating BSs. Therefore, it is important to find solutions which decrease the overhead, such as to limit the number of BSs involved in joint processing by forming clusters.

A simulation study of the performance over the cluster area of three joint processing schemes for the downlink is presented in [9]. Each one of the schemes introduces various amounts of overhead, in terms of required channel knowledge at the transmitter side, inter-base information exchange and feedback from the users. In practice, the cluster of BSs may dynamically adapt the joint processing scheme based on, e.g., user requirements and available system resources.

In this paper, we focus on user requirements and evaluate the impact of the joint processing schemes in [9] on the utility of the three typical classes of Internet applications, hard realtime, adaptive, and elastic applications. The qualitative results show that the potential performance gain offered by joint processing is highly dependent on the application type. For hard real-time applications, joint processing can bring large performance gains, but only if the increase in data rate is large enough for the utility of a user to go from zero to one. A smaller increase does not improve the utility for hard realtime applications and only adds unnecessary overhead. For adaptive applications, joint processing increases utility. Even small increases in data rate result in higher utility, until the point where the maximum utility is reached. After that point there is no use to increase the data rate further. For elastic applications, the impact of joint processing on utility is less significant, since the data rate achieved with single base station assignment is large enough to increase utility to a high level.

The paper is structured as follows: In Section II, utility functions for Internet applications are discussed and we describe the utility functions used for the simulations. A brief overview of the joint processing schemes is given in Section III. For more details about the joint processing schemes the reader is referred to [9]. In Section IV, numerical results of utility are presented and analyzed. The conclusions of the paper are summarized in Section V.

\section{UTILITY FUNCTIONS}

In this section, utility functions for hard real-time, adaptive, and elastic applications are described in general and the specific utility functions that are used for the simulations are presented. The specific utility functions used are equivalent to the ones presented in [3]. For comparison purposes, we have also included a linear utility function, as shown in Fig. 1. 


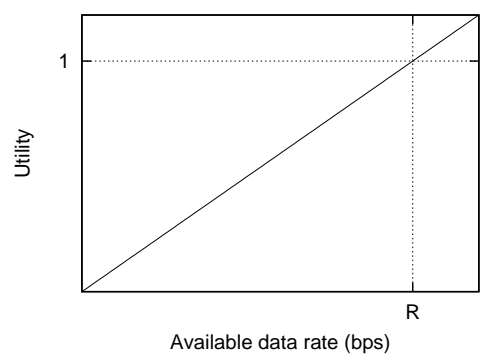

Fig. 1. Linear utility

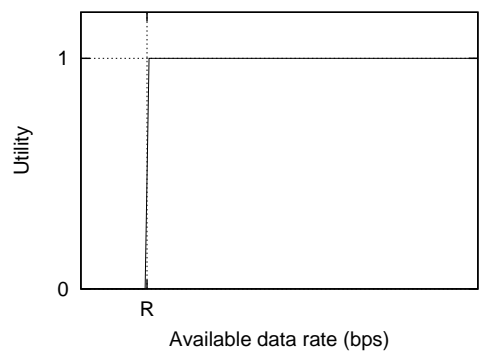

Fig. 2. Utility of real time applications

Assume an application with linear utility. The utility is then proportional to the data rate, for any data rate.

Hard real-time applications, such as speech, require a constant data rate at a certain level which is illustrated by the utility function in Fig. 2. For hard real-time applications, the following utility function for data rate, $r$, is used

$$
u(r)=\frac{\operatorname{sgn}(r-R)+1}{2}
$$

where $r$ is the available data rate and $R$ is the data rate required to maximize utility (to 1 ).

The utility function for adaptive applications, such as streaming media and on-line gaming, shown in Fig. 3, is more or less similar to the one for hard real-time applications, but smoother. The utility function used for data rate for adaptive applications is

$$
u(r)=\frac{1}{1+(1 / \epsilon-1)^{(1-2 r / R)}}
$$

where $R$ is the data rate required to maximize utility (to $1-\epsilon$ ). The smaller the value of $\epsilon$, the steeper the curve. We use $\epsilon=0.01$, which is the same value as in [3].

For elastic applications, such as file transfer and e-mail, the higher the data rate the better, but, as shown in Fig. 4, the utility gain is higher at low data rates. Logarithm functions are commonly used for the utility function of elastic applications [1], [3]. The following utility function is used for data rate for elastic applications

$$
u(r)=\frac{\ln (r+1)}{\ln (R+1)}
$$

where $R$ is the data rate required for a utility value of 1 . Elastic applications can, in contrast to hard real-time and

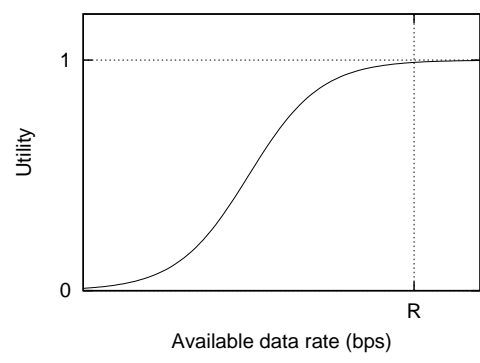

Fig. 3. Utility of adaptive applications

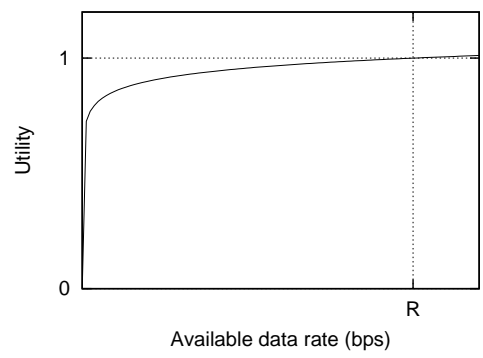

Fig. 4. Utility of elastic applications

adaptive applications, use an even higher data rate, but with only marginally increased utility. The value of $\mathrm{R}$ could, for example, illustrate the maximum data rate that a user pays for.

\section{JOINT PROCESSING SCHEMES}

This section gives a brief overview of the considered joint processing schemes for the downlink of a cluster of BSs. A more extensive description is provided in [9]. The joint processing schemes establish different degrees or stages of joint processing between the BSs in the cluster. Linear precoding obtained using zero-forcing is considered and the available transmission power at each BS is assumed to be limited by a maximum value. For simplicity, equal user power allocation is applied. The joint processing schemes require strong synchronization mechanisms to ensure coherent reception at the user.

\section{A. Centralized joint processing}

In the centralized joint processing (CJP) scheme, global CSI is available at the transmitter side, and the BSs included in the cluster jointly perform the power allocation and the design of the transmit beamformers. This scheme relies on a central unit, which could be located in one of the BSs or in a dedicated network node. The drawback with the CJP scheme is that a large overhead is introduced due to the required information exchange between the BSs and the central unit.

\section{B. Partial joint processing}

The partial joint processing (PJP) scheme is a particular case of the CJP scheme. This scheme defines different degrees or stages of joint processing between BSs. Joint processing degrees are obtained arranging an active set or subset of BSs 


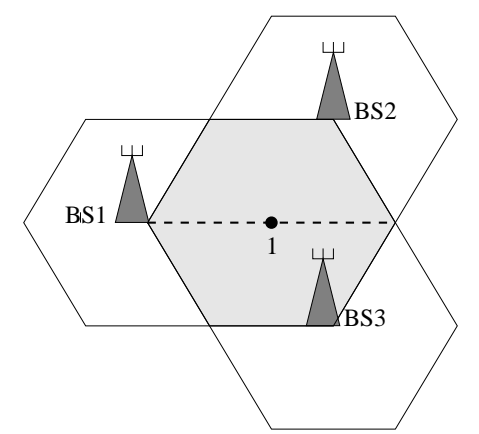

Fig. 5. Cluster area under consideration. The normalized distance (distance/radius) equal to 1 from BS1 is indicated.

for each user in the cluster area, based on a channel gain threshold value. Hence, a user only receives its data from the subset of BSs included in its active set. From the system point of view, three benefits are provided: feedback reduction (users only feed back channels with an acceptable quality), lower inter-base information exchange (user data is only needed in the BSs included in its active set) and efficient distribution of power (power is saved from poor quality channels). However, this joint processing scheme introduces multi-user interference in the system, since less CSI is available at the central unit to design the transmit beamformers.

\section{Distributed joint processing}

In the distributed joint processing scheme (DJP), BSs are only aware of their local CSI. Therefore, the transmit beamforming design and power allocation are locally implemented at each BS (distributed), but the user may receive its data from several BSs (joint processing) depending on its given channel conditions. This scheme requires a multi-base scheduling technique to assign users to BSs under a joint processing assumption [9].

\section{NUMERICAL RESULTS}

In this section, qualitative simulation results of application utility are presented. The aim is to determine to what extent joint processing can be used to increase application utility. The simulations are conducted under commonly used simulation assumptions. A cluster of $3 \mathrm{BSs}$, each one equipped with an array of 3 antennas, is simulated. The cluster radius and height are 500 and 433 meters, respectively. The channel vector between the $m$ th user and the $k$ th BS is modeled as $\boldsymbol{h}_{m k}=\boldsymbol{h}_{m k}^{\prime} \sqrt{\gamma_{s} \gamma_{p}}$, where the shadow fading is a random variable described by a log-normal distribution, $\gamma_{s} \sim \mathcal{N}(0,8 d B)$, the pathloss follows the 3GPP Long Term Evolution (LTE) model, $\gamma_{p}(d B)=148.1+37.6 \log _{10}\left(r_{m k}\right)$, and $\boldsymbol{h}^{\prime}{ }_{m k}$ includes the small-scale fading coefficients, which are i.i.d. complex Gaussian values according to $\mathcal{C N}(0,1)$. The system SNR is $15 \mathrm{~dB}$ (reference value for one user at the cell edge), which implies an interference limited system. The cluster area is illustrated in Fig. 5. In the simulations presented in this paper, 6 users are uniformly distributed within about 30 meters around eight equally distanced points along the

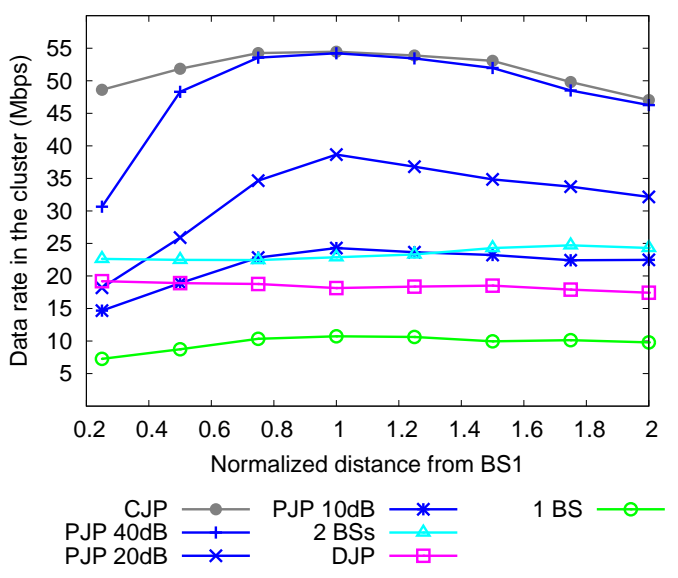

Fig. 6. Data rate of joint processing schemes vs. normalized distance (distance/radius) from BS1

dashed line from BS1 towards BS2 and BS3. The user data rate $\left(\log _{2}\left(1+\mathrm{SINR}_{m}\right) * \mathrm{BW}\right.$, where $\mathrm{SINR}_{m}$ is the signal to interference plus noise ratio of the $m$ th user, and $\mathrm{BW}$ is the bandwidth of the signal, $1 \mathrm{MHz}$ ) is evaluated and averaged over 500 independent channel realizations, following the simulation methodology of [9]. In the simulations, 3 active set threshold values $(10,20$ and $40 \mathrm{~dB})$ are considered for the PJP scheme (PJP 10dB, PJP 20dB, and PJP 40dB, respectively). In addition to the CJP, PJP and DJP schemes, results for the conventional case in which each user receives its data from a single BS are included as a base-line (1 BS). In the $1 \mathrm{BS}$ case, each BS is serving 2 users, and the beamformers are locally designed. Finally, the particular case of the PJP scheme where joint processing of the two BSs to which the user have the best channel conditions is also included ( $2 \mathrm{BSs}$ ).

Fig. 6 shows the total data rate of the 6 users in the cluster vs. normalized distance (distance/radius) from BS1 towards the other BSs along the line in Fig. 5. Note that these results are proportional to linear utility presented in Section II. All joint processing schemes increase the data rate as compared to $1 \mathrm{BS}$. The highest data rates are achieved with CJP. The data rates for PJP $40 \mathrm{~dB}$ are almost as high, since a threshold value of $40 \mathrm{~dB}$ allows cooperation of close to $3 \mathrm{BSs}$, on average.

\section{A. Hard real-time applications}

In Fig. 7, total utility of all 6 users vs. normalized distance (distance/radius) from BS1 is illustrated for hard real-time applications. The data rate requirement, corresponding to $\mathrm{R}$ in the utility function, is $3 \mathrm{Mbps}$. The particular value of $3 \mathrm{Mbps}$ was chosen, since it illustrates an interesting operating point. Total utility is increased with all the joint processing schemes as compared to $1 \mathrm{BS}$, for which total utility is zero at all distances. Total utility reaches the maximum value of 6 for CJP, PJP 40dB, and 2 BSs at all distances from BS1, which results in overlapping curves. For the first distance point, the maximum total utility is not reached with PJP 20dB and PJP $10 \mathrm{~dB}$. Total utility varies between 1 and 6 with DJP, depending on the distance from BS1. 


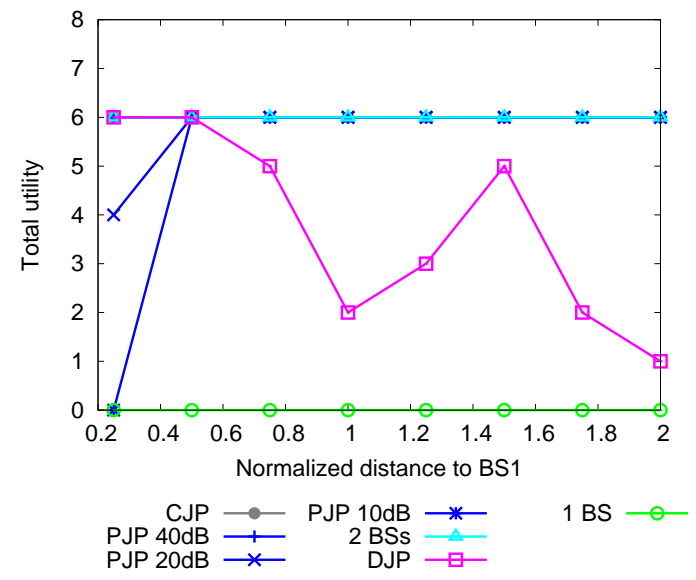

Fig. 7. Total utility for hard real-time applications with required data rate $\mathrm{R}=3 \mathrm{Mbps}$ vs. normalized distance (distance/radius) from BS1

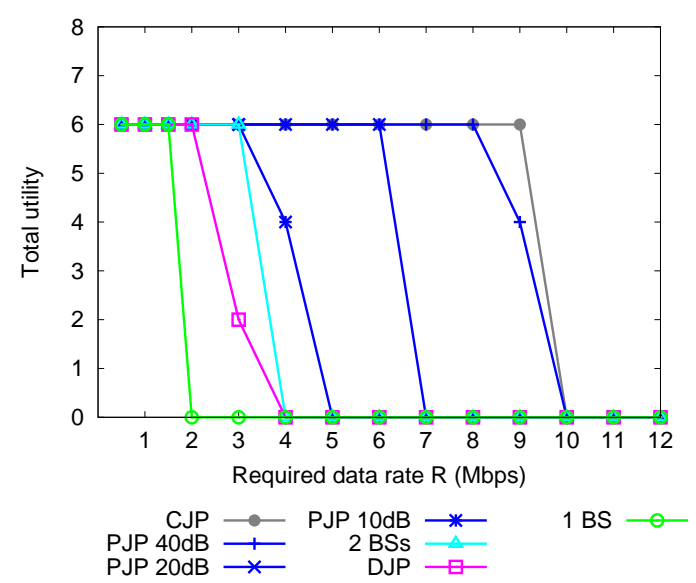

Fig. 8. Total utility for hard real-time applications vs. required data rate R, at normalized distance (distance/radius) equal to 1 from BS1

In Fig. 8, the impact of data rate requirement is illustrated for the point at normalized distance 1 from BS 1, indicated in Fig. 5. Higher total utility is maintained for high data rates for all joint processing schemes than for $1 \mathrm{BS}$. The maximum total utility is provided for a joint processing scheme until the required data rate of the hard real-time applications is too high in relation to the data rate provided with the joint processing scheme. For example, total utility is high up until $9 \mathrm{Mbps}$ for CJP.

The utility of hard real-time applications is improved with joint processing when the increase in data rate is large enough for the utility of a user to go from zero to one. If the increase in data rate is smaller or the data rate requirement is already satisfied, then there is no gain of joint processing for hard real-time applications.

\section{B. Adaptive applications}

For adaptive applications with data rate requirements of $3 \mathrm{Mbps}$, high total utility is reached for all joint processing schemes, as shown in Fig. 9. Also in Fig. 10, for the required

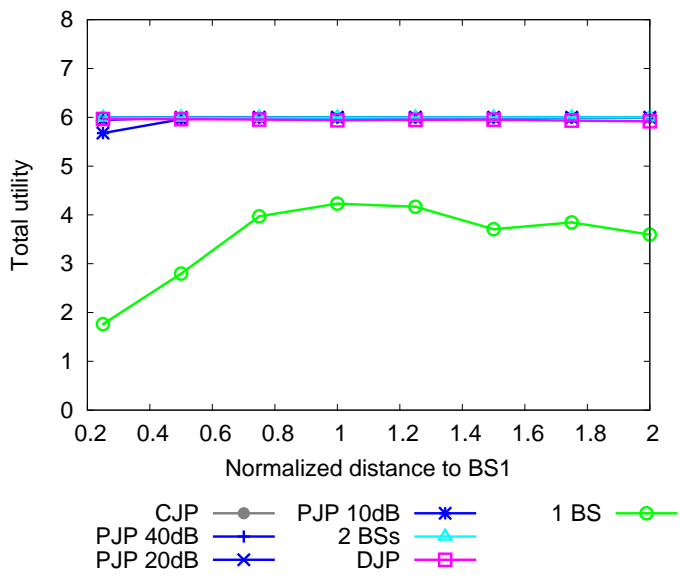

Fig. 9. Total utility for adaptive applications with required data rate $\mathrm{R}=3 \mathrm{Mbps}$ vs. normalized distance (distance/radius) from BS1

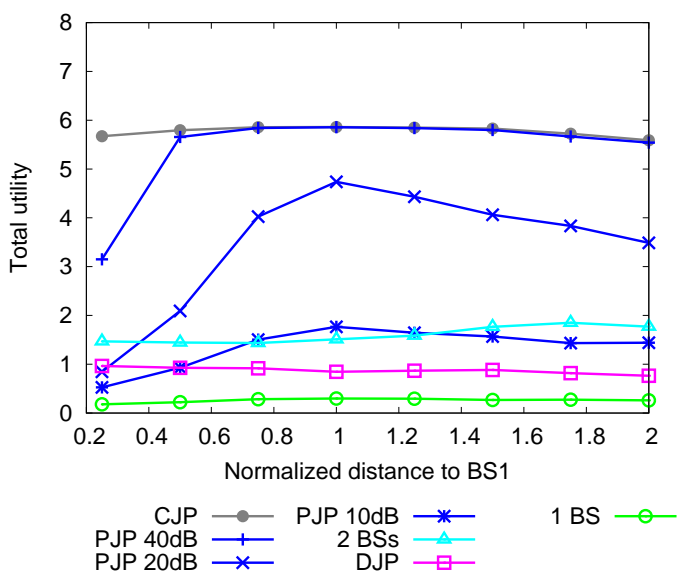

Fig. 10. Total utility for adaptive applications with required data rate $\mathrm{R}=10 \mathrm{Mbps}$ vs. normalized distance (distance/radius) from BS1

data rate $10 \mathrm{Mbps}$, the joint processing schemes provide higher total utility than $1 \mathrm{BS}$. Here, the differences between the joint processing schemes are large. The highest total utility is reached with CJP and PJP $40 \mathrm{~dB}$. The other joint processing schemes provide a much lower total utility. The impact of data rate requirement is illustrated in Fig. 11 for the point at normalized distance 1 from BS1.

For adaptive applications that require a relatively high data rate, the utility is significantly improved with the increased data rate provided with joint processing. In some cases, depending on the exact shape of the utility function, when the offered data rate is very low and the utility of the adaptive application increases very slowly, joint processing could be used to ensure a minimum level of data rate.

\section{Elastic applications}

For elastic applications, there are only small differences between the joint processing schemes. Also for $1 \mathrm{BS}$ high total utility is reached, as illustrated in Fig. 12 and 13. When the required data rate is $3 \mathrm{Mbps}$, as shown in Fig. 12, total 


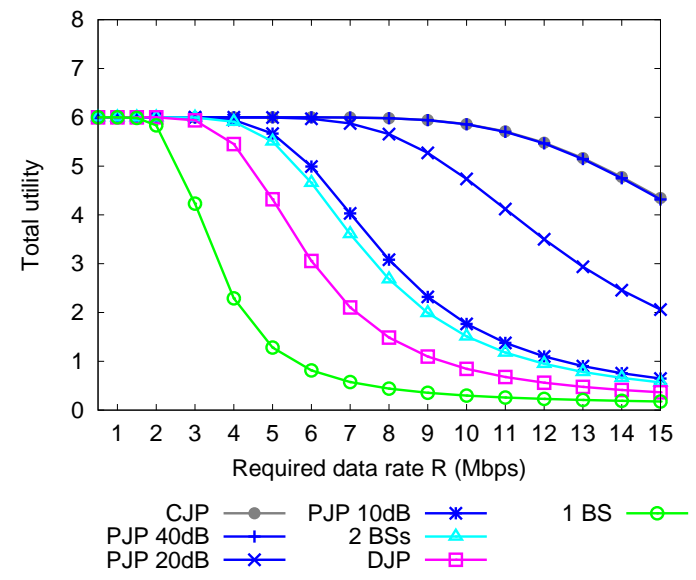

Fig. 11. Total utility for adaptive applications vs. required data rate $\mathrm{R}$ at normalized distance (distance/radius) equal to 1 from BS1

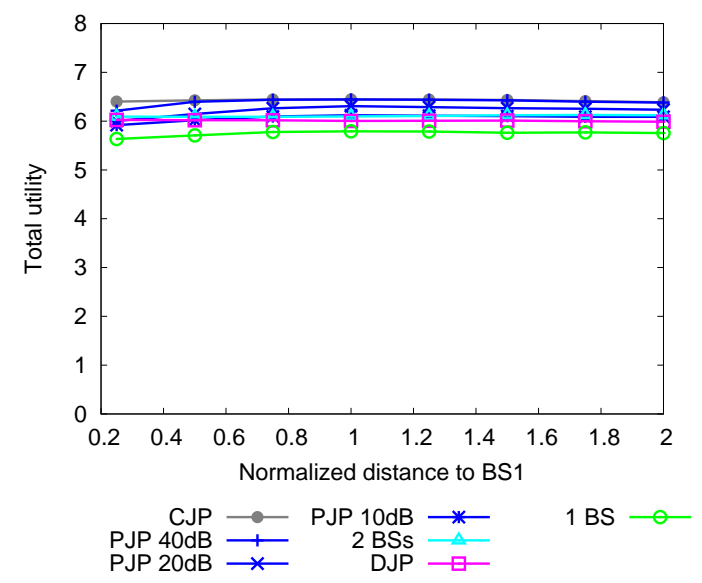

Fig. 12. Total utility for elastic applications with required data rate $\mathrm{R}=3 \mathrm{Mbps}$ vs. required data rate $\mathrm{R}$ at normalized distance (distance/radius)

utility is highest for CJP and lowest for 1 BS. The difference between the the highest and the lowest values is however low, less than 1 . Fig. 13 shows that total utility degrades slowly with increasing required data rate.

Joint processing would not be efficient to improve utility of elastic applications, since the utility achieved with joint processing is only negligibly higher than with $1 \mathrm{BS}$. For an elastic application that would also have a requirement on minimum data rate, below which the utility is zero, joint processing might be useful to ensure the minimum data rate.

\section{CONCLusions}

In this paper, the impact on the utility of typical Internet applications, hard real-time, adaptive, and elastic applications, is evaluated for three joint processing schemes. The qualitative results indicate that the impact of joint processing on utility is highly dependent on the application type. The utility of hard real-time applications is improved with joint processing only in cases when the increase in data rate is large enough for the

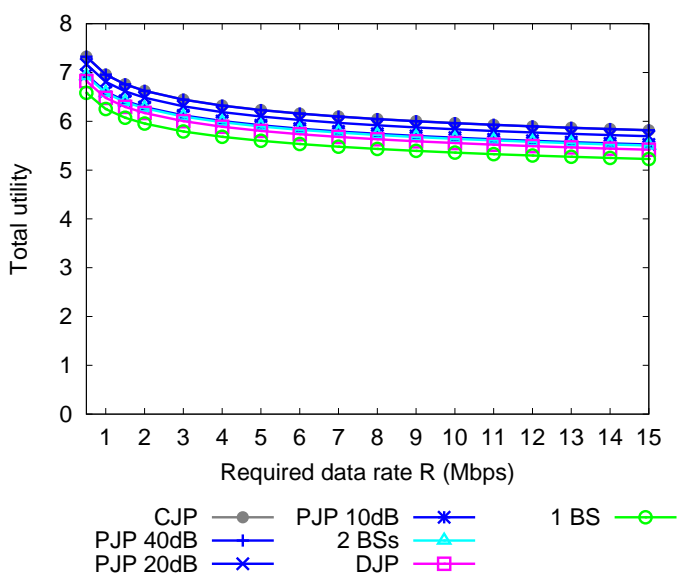

Fig. 13. Total utility for elastic applications vs. required data rate $\mathrm{R}$ at normalized distance (distance/radius) equal to 1 from BS1

utility for a user to change from zero to one. Adaptive applications gain from joint processing and also relatively small increases in data rate improve utility. For elastic applications, joint processing only brings small improvements in utility and, therefore, it is more efficient to allocate the resources needed for joint processing to other application types.

\section{ACKNOWLEDGMENT}

This work is part of Project 2009-4555 Dynamic Multipoint Wireless Transmission which is financed by the Swedish Research Council.

\section{REFERENCES}

[1] L. Breslau and S. Shenker, "Best-effort versus reservations: a simple comparative analysis," Proceedings of the ACM SIGCOMM '98 conference on Applications, technologies, architectures, and protocols for computer communication, 1998.

[2] Z. Jiang, Y. Ge, and Y. G. Li, "Max-utility wireless resource management for best-effort traffic," IEEE Transactions on Wireless Communications, vol. 4, pp. 100-111, January 2005.

[3] L. Shi, C. Liu, and B. Liu, "Network utility maximization for tripleplay services," Computer Communications, vol. 31, pp. 2257-2269, June 2008.

[4] M. Kassar, B. Kervella, and G. Pujolle, "An overview of vertical handover decision strategies in heterogeneous wireless networks," Computer Communications, vol. 31, pp. 2607-2620, June 2008.

[5] J. Sachs, M. Prytz, and J. Gebert, "Multi-access management in heterogeneous networks," Wireless Personal Communications, vol. 48, pp. 7 32, January 2009

[6] S. Shenker, "Fundamental design issues for the future Internet," IEEE Journal of Selected Areas in Communication, vol. 13, pp. 1141-1149, September 1995.

[7] P. Marsch and G. Fettweis, "On multicell cooperative transmission in backhaul-constrained cellular systems," Annals of Telecommunication, vol. 63 , pp. 253-269, June 2008 .

[8] V. Jungnickel et al., "Coordinated multipoint trials in the downlink," Proc. 5th IEEE Broadband Wireless Access Workshop (BWAWS), colocated with IEEE GLOBECOM 2009, November 2009.

[9] C. Botella, T. Svensson, X. Xu, and H. Zhang, "On the performance of joint processing schemes over the cluster area," IEEE Vehicular Technology Conference Spring 2010, May 2010.

[10] 3GPP, "TR 36.814-900 3rd Generation Partnership Project; Technical Specification Group Radio Access Network; Further Advancements for E-UTRA Physical Layer Aspects (Release 9);”’ March 2010. 